\title{
The mediation of interleukin-17 and chemokine ligand 2 in pelvic pain of experimental autoimmune prostatitis
}

\author{
XIAODONG LIU, SHICHENG FAN, MINGXING ZHENG, JIANHENG CHEN, JIANHUA ZHANG and HAO LI
}

Department of Urology, The First Affiliated Hospital of Kunming Medical University, Kunming, Yunnan 650032, P.R. China

Received October 12, 2016; Accepted January 13, 2017

DOI: 10.3892/etm.2017.4448

\begin{abstract}
The present study aimed to determine the expression and mediation of interleukin-17 (IL-17) and chemokine ligand 2 (CCL2) in a rat model with experimental autoimmune prostatitis (EAP). A total of 44 Sprague Dawley (SD) rats were used in the present study. Of these, a total of 20 two-month-old SD rats were randomly divided into a normal control $(n=10)$ and a model group (EAP group, $n=10$ ). The remaining 24 two-month old SD rats were treated in the same way as EAP rats and subsequently randomly divided into a tacrolimus group $(n=8)$, a celecoxib group $(n=8)$ and a normal saline (NS) control group $(n=8)$. Rats in the EAP and normal control groups underwent the Von Frey filaments behavioral test; rats in the tacrolimus, celecoxib and normal saline groups received a pain test following intervention treatment. Prostate tissues of SD rats in each group were harvested for reverse transcription-quantitative polymerase chain reaction (RT-qPCR) and western blot analysis to observe the expression of IL-17 and CCL2. In the pain-reaction test, the occurrence of abnormal pain in the EAP group was significantly higher compared with the control group $(\mathrm{P}<0.001)$. The celecoxib group experienced a significant decrease in pain at day 10 compared with the NS group $(\mathrm{P}<0.01)$, while the decrease in pain experienced by the tacrolimus group was only significant at day $30(\mathrm{P}<0.001)$ and the pain experienced by the NS group decreased slightly over this same period. Results of RT-qPCR and western blot analysis indicated that, compared with the control group, the expression of IL-17 and CCL2 in the prostate tissue of EAP rats was significantly upregulated 50 days following modeling $(\mathrm{P}<0.05)$. On day 30 following intervention, the expression of IL-17 and CCL2 in the prostate of rats in the tacrolimus and celecoxib groups was significantly downregulated compared with the NS group $(\mathrm{P}<0.05)$. Therefore, the results of the current study
\end{abstract}

Correspondence to: Dr Shicheng Fan, Department of Urology, The First Affiliated Hospital of Kunming Medical University, 295 Xichang Kunming Road, Kunming, Yunnan 650032, P.R. China E-mail: fjtf54@163.com

Key words: prostatitis, interleukin-17, chemokine ligand 2, mechanical pain sense demonstrate that IL-17 and CCL2 serve a vital role in the morbidity of the experimental autoimmune prostatitis and may also have a mediation effect on pelvic pain associated with chronic prostatitis.

\section{Introduction}

Chronic prostatitis (CP) is a frequently occurring disease of the male urogenital system. It has a complicated pathogenesis and its morbidities differ depending on the region it is reported in. The incidences of prostatitis reported in different studies vary due to the application of different epidemiological investigation methods and population structures. In the USA, the incidence of prostatitis among males aged 20 to 79 years old was reported as 2.2-16\% in 1996 (1,2). In Europe, the incidence among males aged 20 to 59 years old was reported as $14.2 \%$ in 1990 (2), and in Asia, the incidence among males aged from 20 to 79 years old was reported to be $2.7-8.7 \%$ in $2000(3,4)$. According to the dynamic medicine investigation database of America, $\mathrm{CP}$ accounts for $>8 \%$ of all patients visiting the Urologic Surgery Clinic (5), but reaches up to $33 \%$ in China (6). According to the classification of prostatitis of the National Institutes of Health (NIH) of America, type III prostatitis (chronic prostatitis/chronic pelvic pain syndrome, CP/CPPS), also known as the chronic non-bacterial prostatitis/chronic pelvic pain syndrome, accounts for $>90 \%$ of $\mathrm{CP}$ cases (7). However, the pathogenesis of CP/CPPS remains unknown as its etiology is complicated and may be associated with the systemic inflammatory and autoimmune mechanism (8). Some reports suggest that $\mathrm{CP} / \mathrm{CPPS}$ is caused by multiple etiological factors; some have reported that it is attributable to some undistinguishable disease with the same or similar clinical presentation, even though they have been cured (9). Currently, many scholars believe that the combined action of pathogenic infection, inflammation, abnormal pelvic floor neuromuscular activity and immunologic mechanism is the major cause of CP/CPPS $(10,11)$. According to the number of white blood cells in the expressed prostatic secretion of patients, the syndrome may be further divided into two subtypes: IIIA (inflammatory CPPS) and IIIB (non-inflammatory CPPS) (12). Considering the prevalence of CPPS can reach $15 \%$ in males, understanding and investigating its pathogenesis is crucial (13). Experimental autoimmune prostatitis (EAP) refers to a non-infection autoimmunity-driven rat model of CPPS; following the subcutaneous injection of prostate antigen (PAG) and the induction 
of adjuvant, pain occurs in rats allowing the pain sense test to be completed.

Interleukin-17 (IL-17) is a novel pro-inflammatory cytokine, a glycoprotein that contains terminal signal peptide and is secreted by a group of independent cluster of differentiation (CD) $4+\mathrm{T}$ cell subsets $(14,15)$. It has been demonstrated that IL-17 is associated with the occurrence and development of various types of disease; its expression increases in a number of autoimmune diseases including rheumatoid arthritis, systemic lupus erythematosus and autoimmune thyroid disease (16). It has been suggested that IL-17 may serve a vital role in autoimmune diseases and animal experiments verify that a number of different types of autoimmune disease in mice may be treated by inhibiting IL-17 expression (17). Previously, type III prostatitis was thought to be a type of autoimmune disease (11), thus it is of great importance to investigate IL-17 expression in the prostate.

It has previously been demonstrated that chemotactic factors serve a vital role in the development of CPPS (18). Chemokine ligand 2 (CCL2) has a chemotaxis and activation effect on inflammation-related natural killer cells, mononuclear macrophages, immunoreaction related $\mathrm{T}$ cells and dendritic cells, which may stimulate the accumulation inflammatory cells in tissue lesions (19), thus causing inflammation in cells and tissues.

The experiments in the current study were performed to analyze the expression of IL-17 and CCL2 in the prostate of EAP rats in addition to assessing the association between IL-17 and CCL2 expression. Pelvic pain in EAP rats was assessed and rats were subjected to the pain behavioral test. Following intervention using tacrolimus and celecoxib, the pain behavioral test was performed again and changes in the expression of IL-11 and CCL2 in the prostate were detected. The results of the current study may improve understanding regarding CPPS pathogenesis. The effect of cytokines and chemotactic factors that mediate the prostate and thus, derive the pelvic pain immunologic adaptive response should be further studied.

\section{Materials and methods}

Animals. A total of 44 two-month old male specific pathogen free (SPF) Sprague Dawley (SD) rats weighing 220 $18 \mathrm{~g}$ and 20 four-month-old SPF SD rats weighing 350 $\pm 20 \mathrm{~g}$ were purchased from the animal experimental center of Kunming Medical University in China (Kunming, China). All rats were raised in individually ventilated cages in the animal house of Kunming Institute of Zoology in the Chinese Academy of Sciences (Kunming, China). The room temperature was $18-22^{\circ} \mathrm{C}$, the humidity was kept at $50 \%$, rats experienced a 12-h light/dark cycle, and all rats had ad libitum access to food and water. All of the experimental protocols in the present study were approved by the Animal Care and Use Committee at Kunming Institute of Sciences (Yunnan, China).

Modeling. The 20 two-month-old SD rats were sacrificed by cervical dislocation following intraperitoneal injection of 10\% chloral hydrate (1011268; Shanghai Rongbai Biological Technology Co., Ltd., Shanghai, China). The complete prostate tissue was harvested from the rats, weighed, and cut into sections. The tissue was subsequently transferred to a glass homogenizer in an ice bath at $0^{\circ} \mathrm{C}$ for $15 \mathrm{~min}$. The obtained homogenate was centrifuged at $5,738 \mathrm{x} \mathrm{g}$ at $4^{\circ} \mathrm{C}$ for $30 \mathrm{~min}$, followed by the extraction of PAG as per the method proposed by Donadio and Depiante-Depaoli (20). Briefly, the protein concentration of PAG was detected using a bicinchoninic acid (BCA) protein assay kit (PC0020-50; Beijing Solarbio Science and Technology Co., Ltd., Beijing, China) and subsequently diluted to $60 \mathrm{mg} / \mathrm{ml}$ solution with PBS. To induce EAP, 34 SD rats (two-month old and weighing $220 \pm 18 \mathrm{~g}$ ) were administered with $1 \mathrm{ml}$ mixed emulsion PAG and complete Freund's adjuvant (F5881; Sigma-Aldrich; Merck KGaA, Darmstadt, Germany; 1:1) by subcutaneous injection at multiple points (under the skin of pelvis area of the lower abdomen and the bilateral shoulders) on days $0,7,14$ and 28. A total of $10 \mathrm{SD}$ rats in the normal group (NG; two-months old and weighing $220 \pm 18 \mathrm{~g}$ ) were administered $1 \mathrm{ml}$ normal saline by subcutaneous injection on the same days.

Pain sense test. On days 5, 10, 20, 30, 35, 40 and 45 following the PAG injection, the Von Frey filament behavioral test was performed to detect the pain sense in the pelvic area of rats in the model group and control group. The Von Frey filament behavioral test was developed in the late 19 century; sets of filaments with different hardness were designed to evaluate sensitivity to pain when touched $(21,22)$. The filament is a piece of non-invasive experimental equipment used to assess the mechanical pain sense in addition to the touch threshold (21). As animal models are unable to express their discomfort directly, the results of this experiment are assessed through a series of behaviors including sudden contraction of the abdomen, immediate licking or scratching of simulated sites and jumping, all of which were defined as positive reactions. The abnormal pain sense test (22) was performed on days 0 , 10,20 and 30 following intervention.

Intervention. A total of 24 EAP rats were divided into three groups $(\mathrm{n}=8)$ and each group was administered either $1 \mathrm{ml}$ tacrolimus (Astellas Pharma Inc., Tokyo, Japan; $0.8 \mathrm{mg} / \mathrm{kg}$ ), $1 \mathrm{ml}$ celecoxib (Pfizer Inc., New York, NY, USA; $40 \mathrm{mg} / \mathrm{kg}$ ) or $1 \mathrm{ml}$ normal saline as a control by intragastric administration once a day for thirty consecutive days.

Reverse transcription-quantitative polymerase chain reaction $(R T-q P C R)$. RT-qPCR was used for the detection of IL-17 and CCL2. TRIzol was used for the extraction of mRNA; tissues specimens were incubated with TRIzol for 5-10 min at room temperature and subsequently centrifuged at $8,263 \mathrm{x} \mathrm{g}$ at $4^{\circ} \mathrm{C}$ for $10 \mathrm{~min}$. cDNA was obtained following reverse transcription; the primer was designed according to sequences; target sequences were amplified using a TIANScript RT kit (cat. no. KR104-02; Tiangen Biotech Co., Ltd., Beijing, China) according to the manufacturer's protocol and the reaction system was constructed for PCR. The housekeeping gene $\beta$-actin was used as the internal control in sample results. The procedures for RT-qPCR were as follows: Firstly, $1 \mu \mathrm{g}$ of total RNA, $2 \mu \mathrm{l}$ of Oligo (dT) and $2 \mu 1$ of Super Pure dNTP were added into a reaction tube and mixed. The reaction mixture was diluted to $14.5 \mu \mathrm{l}$ through the addition of RNase-Free $\mathrm{ddH}_{2} \mathrm{O}$ and heated at $70^{\circ} \mathrm{C}$ for 
$5 \mathrm{~min}$. The mixture was immediately cooled on ice for $2 \mathrm{~min}$, following which the reaction liquid was centrifuged at $51 \mathrm{x} \mathrm{g}$ at room temperature for $10 \mathrm{sec}$ to collect the reaction liquid as some liquid was adhered to the wall following heating. A total of $14.5 \mu \mathrm{l}$ of mixture, $0.5 \mu \mathrm{l}$ of $5 \mathrm{X}$ First-Strand Buffer, $0.5 \mu \mathrm{l}$ of RNasin and $1 \mu \mathrm{l}$ of TIANScrip M-MLV were added. The product was agitated prior to incubation for $10 \mathrm{~min}$ at $25^{\circ} \mathrm{C}$, for $50 \mathrm{~min}$ at $42^{\circ} \mathrm{C}$ and $5 \mathrm{~min}$ at $95^{\circ} \mathrm{C}$. For PCR, the reaction system $(20 \mu \mathrm{l})$ comprised $10 \mu \mathrm{l}$ of SuperReal PreMix Plus, $0.6 \mu \mathrm{l}$ of upstream primer $(10 \mu \mathrm{M}), 0.6 \mu \mathrm{l}$ of downstream primer $(10 \mu \mathrm{M}), 100 \mathrm{ng}$ of cDNA and $0.4 \mu \mathrm{l}$ of ROX Reference Dye and RNase-free $\mathrm{ddH}_{2} \mathrm{O}$ to volume. The primer sequences were as follows: IL-17, forward CAC TGAGGCCAAGGACTT and reverse CGTGGAACGGTT GAGGTA; MCP1, forward CATGCTTCTGGGCCTGCTGT and reverse AGGTGAGTGGGGCGTTAACT. The reaction procedures included pre-degeneration at $95^{\circ} \mathrm{C}$ for $15 \mathrm{~min}$, and 40 cycles of degeneration at $95^{\circ} \mathrm{C}$ for $10 \mathrm{sec}$, annealing at $58^{\circ} \mathrm{C}$ for $30 \mathrm{sec}$ and extension at $72^{\circ} \mathrm{C}$ for $30 \mathrm{sec}$. The quantification of each specimen was repeated three times. Relative quantitative analysis was performed using a fluorescent quantitative PCR amplifier, SuperReal PreMix Plus (SYBR Green; cat. no. FP205-02; Tiangen Biotech Co., Ltd.) and the $2^{-\Delta \Delta \mathrm{Cq}}$ method (23).

Western blot analysis. Western blot analysis was used to detect the gray values of IL-17 and CCL2. Radioimmunoprecipitation assay (RIPA) lysis solution (R0020; Beijing Solarbio Science and Technology Co., Ltd.) was used for cell lysis; the prostate tissues were ground with liquid nitrogen and centrifuged with RIPA lysis solution at $8,263 \times \mathrm{g}$ at $4{ }^{\circ} \mathrm{C}$ for $15 \mathrm{~min}$. The supernatant was collected and protein concentrations were measured using a BCA protein concentration assay kit (PC0020-500; Beijing Solarbio Science and Technology Co., Ltd.) according to the manufacturer's protocol. Proteins were separated by $12 \%$ SDS-PAGE. The amount of protein loaded per lane was: $80 \mu \mathrm{g}$ of IL-17, $160 \mu \mathrm{g}$ of MCP and $20 \mu \mathrm{g}$ of $\beta$-actin (different sample loading standards were formulated for different target proteins). Proteins were transferred onto a polyvinylidene fluoride membrane and blocked with electrophoretic buffer solution for $1.5 \mathrm{~h}$ in an ice bath. Each liter of solution contained the following: $3 \mathrm{~g}$ Tris base (25 Mm; cat. no. 04816100$), 14.4 \mathrm{~g}$ of glycine (cat. no. 04808822; $192 \mathrm{Mm}$ ), $1 \mathrm{~g}$ of SDS (0.1\%; cat. no. 04811030), all MP Biomedicals (Santa Ana, CA, USA). The membrane was subsequently incubated with primary antibodies at $4{ }^{\circ} \mathrm{C}$ overnight against the following: IL-17 (1:500; A0688; ABclonal Biotech Co., Ltd., Lake Bluff, IL, USA), MCP1 (1:600; ab7202; Abcam, Cambridge, UK) and $\beta$-actin (1:10,000; TA-09; Origene Technologies, Inc., Rockville MD, USA). Membranes were washed with PBST (PBST was prepared by adding TWEEN-20 into PBS at a ratio of $1: 5,000)$ three times (10 min each time) and incubated with rabbit-derived antibody (1:3,000; ZB-2301) and mouse-derived antibody (1:3,000; ZB-2305; both Origene Technologies, Inc.) for $1.5 \mathrm{~h}$ at room temperature. Supersignal West Dura Extended Duration Substrate (Pierce; Thermo Fisher Scientific, Inc., Waltham, MA, USA) was used for the detection of enhanced chemiluminescence; exposure, developing and photographic fixing of X-ray film indicated positive bands. The housekeeping gene $\beta$-actin was used as the internal control in sample results. The software used for quantification was GIS 1D analysis software version 4.2 (Tanon, Shanghai, China) (24).

Statistical analysis. Prism version 5.01 (GraphPad Software, Inc., La Jolla, CA, USA) was used for statistical analysis. Two-way analysis of variance was used for data analysis of abnormal tactile sense. $\mathrm{P}<0.05$ was determined to represent a statistically significant difference.

\section{Results}

High expression of IL-17 and CCL2 in prostate tissues of $E A P$ rats. CPPS rat models are currently widely used to study the mechanism between pain and inflammation in human prostatitis (25). EAP rat modeling refers to subcutaneously injecting homologous rat prostate antigens into SD rats to induce prostatitis and paraesthesia of pelvis. The Von Frey filament was adopted for behavioral tests in the present study. Results indicate that the peak value of allodynia in EAP rats occurred 40 days following antigen injection and that the response to tactile alloydnia in EAP rats was significantly higher than that of control rats at all time points $(\mathrm{P}<0.001$; Fig. 1A). Fig. 1 indicates the increase of frequency in response to an abnormal pain stimulus that was exhibited by rats in the EAP group, following the Von Frey test (performed every 10 days) from day 0 to 50, compared with the control group. As the filament strengthened, the response of the control and EAP groups differed significantly at every time point $(\mathrm{P}<0.05$; Fig. 1B and C).

Fifty days following the injection of antigens, prostate tissues of rats in the control group and the EAP group were subjected to RT-qPCR and the expression of IL-17 and CCL2 were compared. Western blot analysis was completed to assess and compare the protein level of IL-17 and CCL2 in prostate tissues of rats. Fig. 2A and B indicate that prostate tissues of rats in the control group and the EAP group exhibited significantly higher levels of IL-17 and CCL2 mRNA, compared with the control $(\mathrm{P}<0.001)$. Fig. $2 \mathrm{C}$ and D demonstrate that prostate tissues of rats in the EAP group exhibited significantly higher levels of IL-17 and CCL2 protein, compared with rats in the control group $(\mathrm{P}<0.001)$. These results indicate that EAP rats experienced pelvic pain. Furthermore, the high expression of IL-17 and CCL2 indicate that both may participate in the occurrence and development of inflammation.

Mediation of IL-17 and CCL2 in pelvic pain of EAP rats. IL-17 and CCL2 exhibited high expression in the prostate tissues of EAP rats, indicating that IL-17 and CCL2 serve an important role in the occurrence and development of inflammation. In order to assess the effect of IL-17 and CCL2 in the mediation of pelvic pain, tacrolimus, celecoxib or normal saline was administered to EAP rats for 30 days. During the intervention treatment, the frequency of abnormal pain reaction demonstrated different degrees of decrease. Fig. 3A (i-iii) demonstrates the differences between each group when each filament was strengthened constantly. Fig. 3A (i) describes the abnormal pain reaction frequency of the mice on days 0 (one day prior to treatment), 10, 20 and 30 after being gavaged with 
A Tactile allodynia
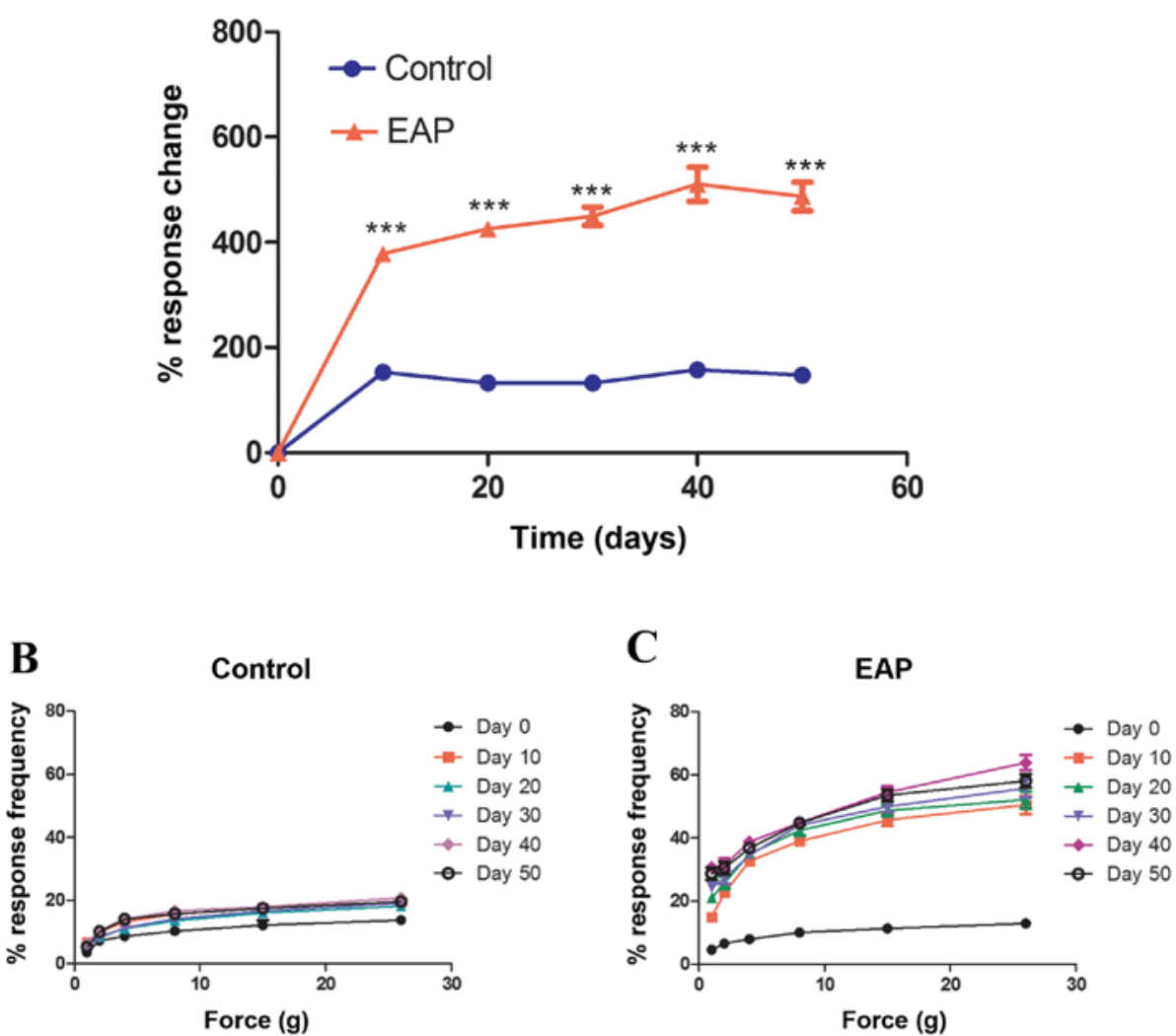

Figure 1. Abnormal pain sense test of EAP rats. (A) Using the reaction frequency of abnormal pain sense prior to antigen injection as the basis, the abnormal pain sense of the control group and the EAP group was assessed every 10 days for 50 days. Using Von Frey filaments with different strength, the (B) control group and (C) EAP group were assessed every 10 days. Data were analyzed using GraphPad Prism software and response changes were assessed by two-way analysis of variance. Data is presented as the mean \pm standard deviation. ${ }^{* * *} \mathrm{P}<0.001$. EAP, experimental autoimmune prostatitis.

A

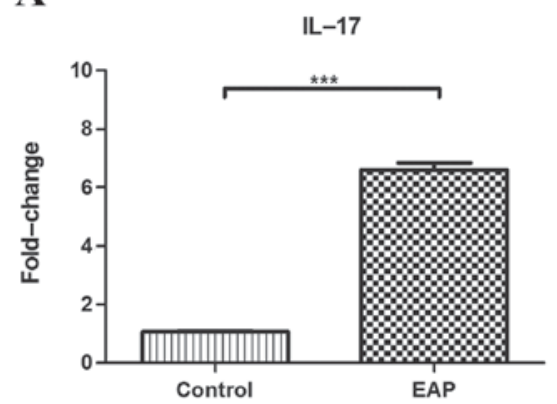

C

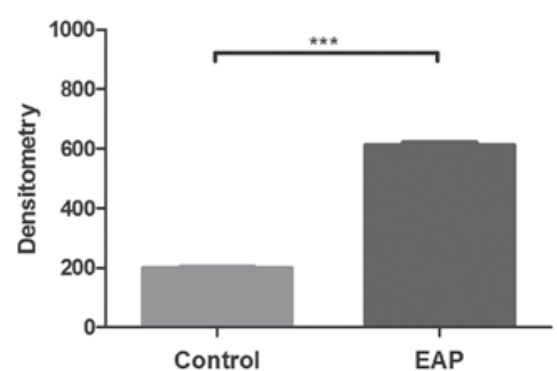

B

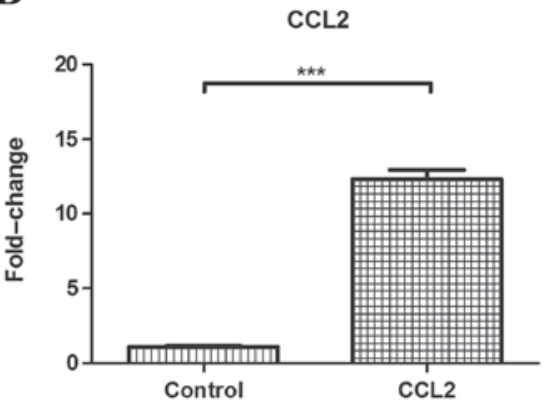

D

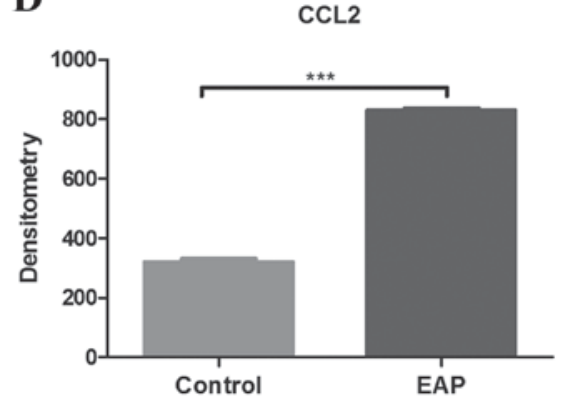

Figure 2. High expression of IL-17 and CCL2 in prostate tissues of rats. Contrastive analysis of quantitative results of prostate tissues of rats in control and EAP groups was obtained using reverse transcription-quantitative polymerase chain reaction for (A) IL-17 and (B) CCL2. Contrastive analysis of gray values of prostate tissues of rats in control and EAP groups was obtained following western blot analysis for (C) IL-17 and (D) CCL2. Data were analyzed using GraphPad Prism software. Data of reverse transcription-quantitative polymerase chain reaction and western blot analysis were assessed using a paired T-test. Data is presented as the mean \pm standard deviation. ${ }^{* * *} \mathrm{P}<0.001$. IL-17, interleukin-17; CCL2, chemokine ligand 2; EAP, experimental autoimmune prostatitis. 
A i

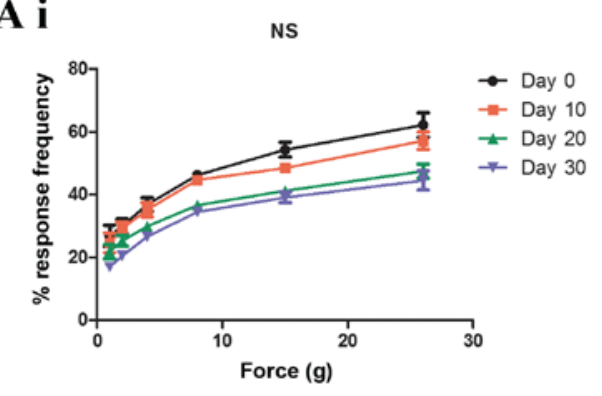

ii

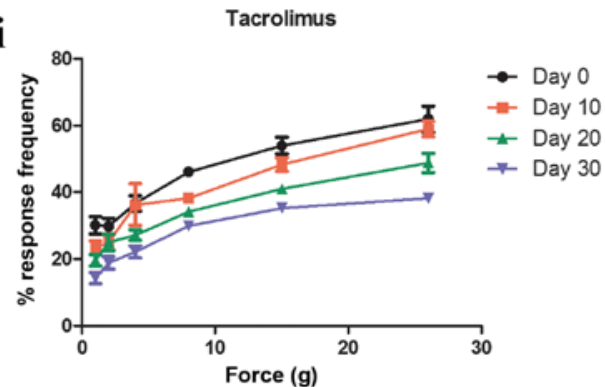

iii

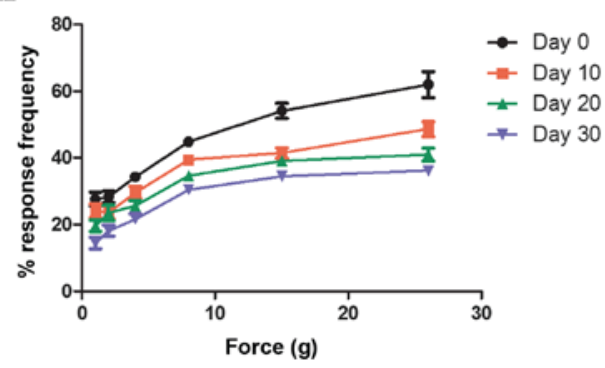

B i

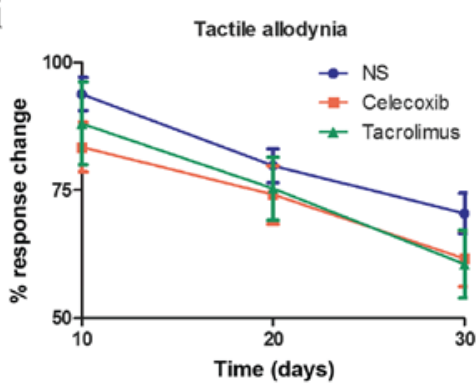

ii

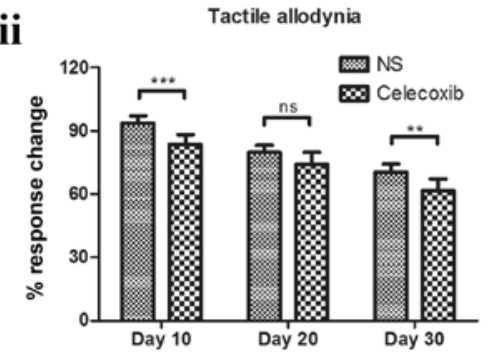

iii

Tactile allodynia
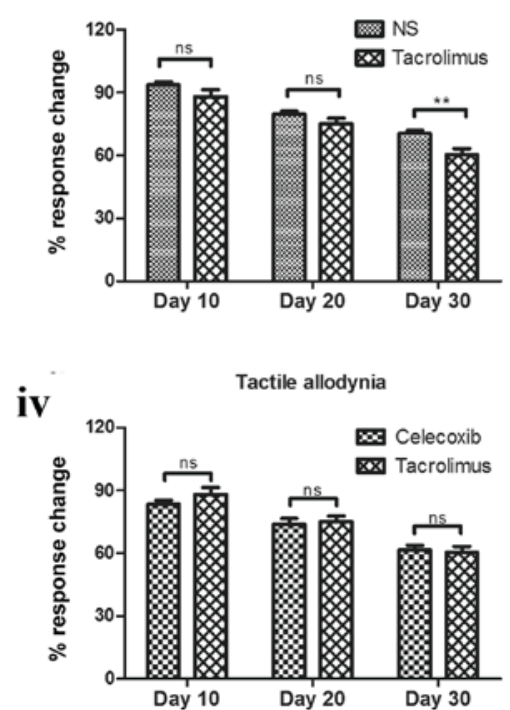

Figure 3. Abnormal pain test of EAP rats following intervention. (A) Following the intervention treatment, abnormal pain tests were completed every 10 days for 30 days to assess changes of reaction frequency for each group under filaments with different strength (A; i) NS, (A; ii) tacrolimus and (A; iii) celecoxib groups. (B; i) Percentage variation between the reaction frequency following 30 days compared with the reaction frequency prior to intervention. Comparisons of the relieved degrees of pelvic pain among the 3 groups every 10 days following intervention for 30 days. No significant difference was observed between the two treatments, tacrolimus and celecoxib. (B; ii) NS vs. celecoxib, (B; iii) NS vs. tacrolimus and (B; iv) celecoxib vs. tacrolimus. Data were analyzed using GraphPad Prism software. Data of abnormal pain tests were tested using two-way analysis of variance. Data is presented as the mean \pm standard deviation. ${ }^{* *} \mathrm{P}<0.01$ and $^{* * *} \mathrm{P}<0.001$. EAP, experimental autoimmune prostatitis; NS, normal saline control.

normal saline; the reaction frequency decreased as time went on. Fig. 3A (ii) shows the abnormal pain reaction frequency of mice on days $0,10,20$ and 30 after being gavaged with tacrolimus; the reaction frequency decreased as time went on. Fig. 3A (iii) demonstrated the abnormal pain reaction frequency of the mice on days 0, 10, 20 and 30 after being gavaged with tacrolimus; the reaction frequency decreased as time went on. Fig. 3B (i) describes results from the Von Frey test. Tests were performed every 10 days following intervention until day 30, and results are presented in Fig. 3B (i). Compared with the NS control group, the frequency of abnormal pain reaction of tacrolimus and celecoxib groups decreased, although this decrease was not significant.
Comparison of the therapeutic effect of tacrolimus and celecoxib on inflammation and pelvic pain of EAP rats. Fig. 3B (ii-iv) presents the results of abnormal pain tests at different time points following intervention, indicating that tacrolimus and celecoxib had lessened pelvic pain in EAP rats. Tacrolimus and celecoxib were able to relieve pelvic pain and control the expression of IL-17 and CCL2 in inflammation. As presented in Fig. 3B (ii-iii), the effectual time of celecoxib group was earlier than that of the tacrolimus group; 10 days following the intervention treatment, the difference between the celecoxib group and the NS control group was significant $[\mathrm{P}<0.001$; Fig 3B (ii)]. However, the difference between the tacrolimus group and the NS control group was significant 
A

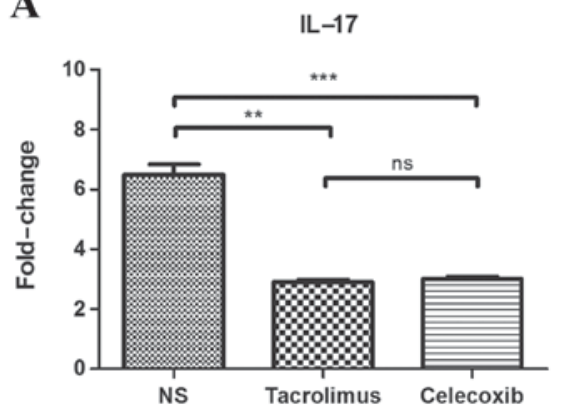

C

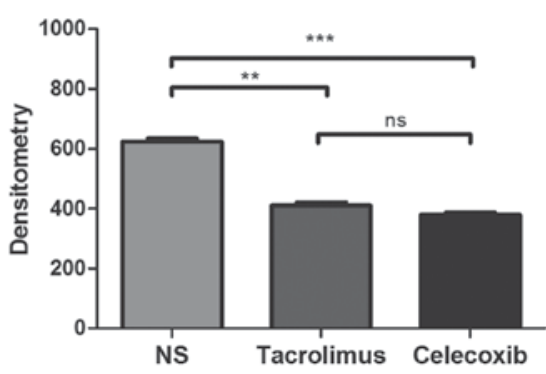

B

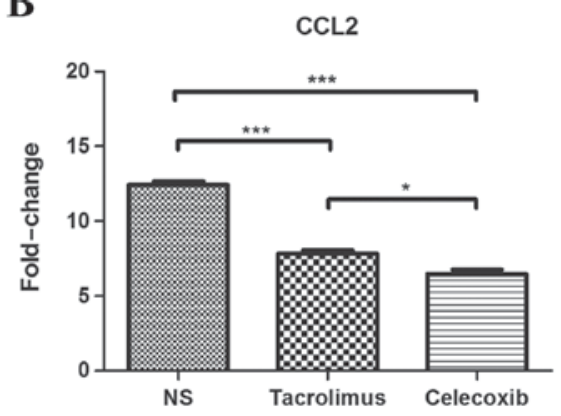

D

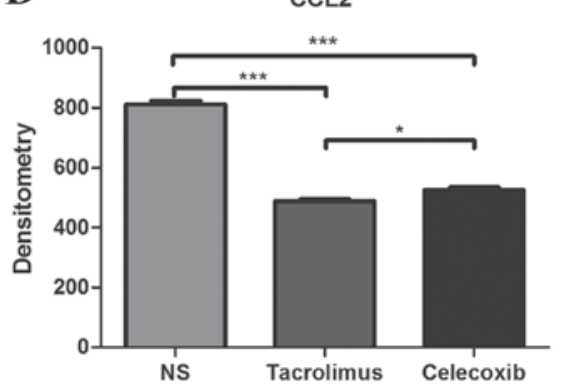

Figure 4. Expression of IL-17 and CCL2 in prostate tissues of rats in each group following intervention. Contrastive analysis of quantitative results of each group was obtained using a reverse transcription-quantitative polymerase chain reaction for (A) IL-17 and (B) CCL2. Contrastive analysis of gray values of each group obtained through western blot analysis for (C) IL-17 and (D) CCL2. Data were analyzed using GraphPad Prism software. Data of reverse transcription-quantitative polymerase chain reaction and western blot analysis were assessed using a paired T-test. Data is presented as the mean \pm standard deviation. ${ }^{*} \mathrm{P}<0.05,{ }^{* *} \mathrm{P}<0.01$ and ${ }^{* * *} \mathrm{P}<0.001$. IL-17, interleukin-17; CCL2, chemokine ligand 2; NS, normal saline control.

30 days following intervention $[\mathrm{P}<0.01$; Fig. 3B (iii)]. In Fig. 3B (iv), the comparison between the celecoxib and tacrolimus groups demonstrated no significant difference between the two treatments at the three time points. Therefore, although the effectual time of celecoxib group was earlier than that of the tacrolimus group, there was no significant difference in therapeutic effect between the two treatments at any time point.

In addition, in prostate tissues, the expression of IL-17 and CCL2 decreased, compared with that of the NS group. As presented in Fig. 4A and B, 30 days following intervention of EAP rats, prostate tissues of rats in each group were removed for RT-qPCR analysis. Results demonstrated that, compared with the NS group, the expression of IL-17 and CCL2 significantly decreased in the tacrolimus and celecoxib groups $(\mathrm{P}<0.01)$. Prostate tissues of rats in each group were obtained 30 days following intervention and subjected to western blot analysis. Results indicated that, compared with the NS group, both tacrolimus and celecoxib decreased the gray value contrast (Fig. 4C and D). This indicates that IL-17 and CCL2 not only serve an important role in occurrence and development of inflammation, but also in the mediation of pelvic pain.

\section{Discussion}

Chronic prostatitis is a disease caused by a number of factors (25) that may lead to discomfort in the groin area, pelvic pain, irritable urination and sexual dysfunction (26). Its histological characterization is the infiltration of multinucleate and mononuclear cells into interstitial connective tissues (27).
However, the pathogenesis and diagnostic criteria for chronic prostatitis remains unclear, thus research in this area is hindered (28).

Good animal models may not only help with the analysis of the pathogenesis of chronic prostatitis and chronic pelvic pain syndrome, but may also provide evidence for the efficiency of different treatment methods. Therefore, appropriate animal models are of great value to understanding and treating this disease $(29,30)$. With the development of immunology and molecular biology, the effect of autoimmune factors on morbidity of chronic prostatitis is receiving greater attention (31). A number of studies $(18,32,33)$ have assessed the association between the immune reaction and pathogenesis of chronic prostatitis to investigate the pathogenesis and pathophysiological process of the disease, meaning that the current model is improving (34). In the antigen-induced animal models of chronic prostatitis, Pacheco-Rupil et al (35) proposed an autoimmune model of antigen-induced inflammation of the prostate of rodents. A mixture of homogenate of the accessory glands of male Wistar rats and the complete Freund's adjuvant was used as an antigen substance and subcutaneously injected into the rats. The results demonstrated that only 3 out of 8 rats (38\%) underwent a prostatitis reaction 21 days following the injection. In a later trial (35), 9 out of 20 rats (45\%) exhibited symptoms of prostatitis 30 days following the injection. A previous study (21) used a model with purified prostate protein (antigen induction) of rats or mice and the complete Freund's adjuvant to construct an autoimmune prostatitis animal model. The method of the current study was simple and highly effective; the mortality rate of animals was low and the model was stable, reliable and had good pathology specificity; the 
pathological changes were similar to the clinical manifestation and the pathogenesis was similar. Therefore, the model may be beneficial in the study of pathogenesis and efficiency for novel treatments for human chronic prostatitis.

Physiologist Maximilien Von Frey was a pioneer of pain research in the late 20th century. He designed the Von Frey filaments, which have differing levels of pressure to assess human's sensitivity to pain when their skin is touched (21). The machine was then named Von Frey filaments or Semmes-Weinstein monofilaments, as a type of non-invasive experimental equipment used to test the mechanical pain sense (point) in addition to the touch threshold. Von Frey filaments are composed of 20 filaments, providing 0.008-300 g tactile stimulus power. During experiments, the size of Von Frey filaments should be selected according to practical situations and the extension length should be adjusted appropriately; filaments are used to stimulate the skin vertically and the curve of filaments indicates complete stress. In short, Von Frey filaments may provide a non-invasive evaluation procedure for cutaneous sensation and the obtained results are objective and are repeatable. During the test of Von Frey filaments, humans may express a direct response. However, in animal model experiments, animals are unable to express discomfort directly. Thus, rats or animals may express a response to stimulation through a series of behaviors. In 2000, Ishigooka et al (36) hypothesized that $\mathrm{CP} / \mathrm{CPPS}$ pain was a type of neuropathic pain and the research focused primarily on mechanical pain sense. The mechanical pain sense measurement of prostatitis animal models was not only used for the research on pathogenesis, but was also extended to pain assessment and intervention treatment.

The emphasis of the present study was to use SD rat models to analyze the immunological mechanism behind CPPS, in addition to assessing the association between pain symptoms of CPPS and the autoimmune effect. Results from a previous study indicated that IL-17 serves a vital role in autoimmune diseases (37). In the current study, IL-17 was highly expressed in the prostate tissues of EAP mice, which was consistent with the above results.

Furthermore, IL-17 may also have an effect on nervous lesions and the development of pain caused by spinal cord injury. A previous animal model study indicated that IL-17 was primarily responsible for the development of pain and a specific antibody for IL-17 may markedly relieve pain (38). The results of the current study demonstrated that the expression of IL-17 increased in prostate tissues of EAP rats, indicating that IL-17 serves a relevant role in autoimmune prostatitis of EAP rats. Following intervention using tacrolimus, the expression of IL-17 in prostate tissues of EAP rats decreased significantly $(\mathrm{P}<0.05)$ and pain in EAP rats was also relieved. In a previous study completed by Murphy et al (39), an anti-IL-17 blocking experiment was able to treat the prostatitis and pain of EAP rats; it was also suggested that the CD25+Forkhead box P3+CD4 T cell axis may be a novel method of treating CPPS and chronic pain.

The results of the current study revealed that the expression of CCL2 increased in the prostate tissues of EAP rats, indicating that CLL2 also participates in the pathogenic process of CPPS. Following intervention using tacrolimus, expression of CLL2 in prostate tissues of EAP rats decreased and the pain of EAP rats was significantly relieved $(\mathrm{P}<0.05)$. A previous study (40) constructed EAP mice models and the expression of CCL2 in prostate tissues of mice was increased; changes in the CPPS pain of mice were also observed and anti-CCL2 blocking was further applied (40). Furthermore, symptoms of pain in CCR2 deficient mice were observed to be significantly relieved, indicating that CLL2 participated in the occurrence of CPPS and the development of pain (40). These results were similar to the conclusions of the present study.

Different factors have a complicated mutual induction or inhibition, interaction effects and participate in the immune adjustment together. The aforementioned research results demonstrate a positive correlation between the expression of IL-17 and CCL2. The current study infers that, although IL-17 may induce CCL2, the increase of CCL2 may also lead to the increase of IL-17. Further studies investigating the correlation and associated mechanisms among different factors may enable the development of novel therapeutic interventions to treat CPPS pain.

In conclusion, the current study investigating the intervention treatment of tacrolimus and celecoxib indicates that both tacrolimus and celecoxib may be used to treat CPPS pain and there is no significant difference in the therapeutic effect of these two treatments. However, considering the high cost of tacrolimus, celecoxib is often selected to treat CPPS. As the conclusions of the current study are based on an experiment involving EAP rats, more reliable data from studies in humans are required. Non-obese diabetic (NOD) mice could be used as an alternative animal model preparation in the future (41). Though SD mice have low risks of death during modeling and their prostate tissues are easy to obtain, there is a difference in the success rate of modeling between SD mice and NOD mice.

\section{Acknowledgements}

The authors of the present study would like to thank Professor Shen Jihong, Dr Zhao Hui and Professor Zhang from the Institute of Zoology of Chinese Academy of Sciences.

\section{References}

1. Collins MM, Stafford RS, O'Leary MP and Barry MJ: Distinguishing chronic prostatitis and benign prostatic hyperplasia symptoms: Results of a national survey of physician visits. Urology 53: 921-925, 1999.

2. Roberts RO, Lieber MM, Rhodes T, Girman CJ, Bostwick DG and Jacobsen SJ: Prevalence of a physician-assigned diagnosis of prostatitis: The Olmsted County study of urinary symptoms and health status Among Men. Urology 51: 578-584, 1998.

3. Tan JK, Png DJ, Liew LC, Li MK and Wong ML: Prevalence of prostatitis-like symptoms in Singapore: A population-based study. Singap Med J 43: 189-193, 2002.

4. Ku JH, Kim ME, Lee NK and Park YH: Influence of environmental factors on chronic prostatitis-like symptoms in young men: Results of a community-based survey. Urology 58: 853-858, 2001.

5. Krieger JN, Riley DE, Cheah PY, Liong ML and Yuen KH: Epidemiology of prostatitis: New evidence for a world-wide problem. World J Urol 21: 70-74, 2003.

6. Guo YH and Li HJ: Prostatitis. People's Military Medical Press, Beijing, p68, 2007.

7. Krieger JN, Nyberg LJ Jr and Nickel JC: NIH Consensus definition and classification of prostatitis. JAMA 282: 236-237, 1999.

8. Quick ML, Wong L, Mukherjee S, Done JD, Schaeffer AJ and Thumbikat P: Th1-Th17 cells contribute to the development of uropathogenic Escherichia coli-induced chronic pelvic pain. PLoS One 8: e60987, 2013. 
9. Zhang K: Guide for the Diagnosis and Treatment of Prostatitis. People's Medical Publishing House, Beijing, 2009.

10. Rowe E, Smith C, Laverick L, Elkabir J, Witherow RO and Patel A: A prospective, randomized, placebo controlled, double-blind study of pelvic electromagnetic therapy for the treatment of chronic pelvic pain syndrome with 1 year of followup. J Urol 173: 2044-2047, 2005.

11. Seethalakshmi L, Bala RS, Malhotra RK, Austin-Ritchie T, Miller-Graziano C, Menon M and Luber-Narod J: 17 beta-estradiol induced prostatitis in the rat is an autoimmune disease. J Urol 156: 1838-1842, 1996.

12. Mahal BA, Cohen JM, Allsop SA, Moore JB, Bhai SF, Inverso G and Dimitrakoff JD: The role of phenotyping in chronic prostatitis/chronic pelvic pain syndrome. Curr Urol Rep 12: 297-303, 2011.

13. Wagenlehner FM, Pilatz A, Bschleipfer T, Diemer T, Linn T, Meinhardt A, Schagdarsurengin U, Dansranjavin T, Schuppe HC and Weidner W: Bacterial prostatitis. World J Urol 31: 711-716, 2013.

14. Cua DJ, Sherlock J, Chen Y, Murphy CA, Joyce B, Seymour B Lucian L, To W, Kwan S, Churakova T, et al: Interleukin-23 rather than interleukin-12 is the critical cytokine for autoimmune inflammation of the brain. Nature 421: 744-748, 2003.

15. Park H, Li Z, Yang XO, Chang SH, Nurieva R, Wang YH, Wang Y, Hood L, Zhu Z, Tian Q and Dong C: A distinct lineage of CD4 T cells regulates tissue inflammation by producing interleukin 17. Nat Immunol 6: 1133-1141, 2005.

16. Langrish CL, Chen Y, Blumenschein WM, Mattson J, Basham B, Sedgwick JD, McClanahan T, Kastelein RA and Cua DJ: IL-23 drives a pathogenic $\mathrm{T}$ cell population that induces autoimmune inflammation. J Exp Med 201: 233-240, 2005.

17. Lubberts E, Koenders MI, Oppers-Walgreen B, van den Bersselaar L, Coenen-de Roo CJ, Joosten LA and van den Berg WB: Treatment with a neutralizing anti-murine interleukin-17 antibody after the onset of collagen induced arthritis reduces joint inflammation, cartilage destruction, and bone erosion. Arthritis Rheum 50: 650-659, 2004.

18. Miller LJ, Fischer KA, Goralnick SJ, Litt M, Burleson JA, Albertsen P and Kreutzer DL: Nerve growth factor and chronic prostatitis/chronic pelvic pain syndrome. Urology 59: 603-608, 2002.

19. Arms L, Girard BM, Malley SE and Vizzard MA: Expression and function of CCL2/CCR2 in rat micturition reflexes and somatic sensitivity with urinary bladder inflammation. Am J Physiol Renal Physiol 305: F111-F122, 2013.

20. Donadio AC and Depiante-Depaoli M: Inflammatory cells and MHC class II antigens expression in prostate during time-course experimental autoimmune prostatitis development. Clin Immuno Immunopathol 85: 158-165, 1997.

21. Schmidt R, Schmelz M, Ringkamp M, Handwerker HO and Torebjörk HE: Innervation territories of mechanically activated C nociceptor units in human skin. J Neurophysiol 78: 2641-2648, 1997.

22. Rudick CN, Schaeffer AJ and Thumbikat P: Experimental autoimmune prostatitis induces chronic pelvic pain. Am J Physiol Regul Integr Comp Physiol 294: R1268-R1275, 2008.

23. Livak KJ and Schmittgen TD. Analysis of relative gene expression data using real-time quantitative PCR and the 2(-Delta Delta C(T)) Method. Methods 25: 402-408, 2001.

24. Fen ZG, Yan WJ, Zhang J and Xu Y: Effects of Calcium Concentration on the Soluble Gq Protein $\alpha$ Subunit in the Photoreceptor Cell of Macrobrachium rosenbergi on Light Adaptation and Dark Adaptation. Zoolog Res 24: 373-376, 2003.
25. Depiante-Depaoli M and Pacheco-Rupil B: Experimental autoimmunity to rat male accessory glands (MAG): Circulating antibodies, immunoglobulins bound to target glands, and immunoglobulins-secreting cells. Am J Reprod Immunol Microbiol 7: 32-38, 1985.

26. Schaeffer AJ: Clinical practice. Chronic prostatitis and the chronic pelvic pain syndrome. N Engl J Med 355: 1690-1698, 2006.

27. John H, Barghorn A, Funke G, Sulser T, Hailemariam S, Hauri D and Joller-Jemelka H: Noninflammatory chronic pelvic pain syndrome: Immunological study in blood, ejaculate and prostate tissue. Eur Urol 39: 72-78, 2001.

28. Pontari MA: Chronic prostatitis/chronic pelvic pain syndrome in elderly men: Toward better understanding and treatment. Drugs Aging 20: 1111-1125, 2003.

29. Orhan I, Onur R, Ilhan N, Ardiçoglu A: Seminal plasma cytokine levels in the diagnosis of chronic pelvic pain syndrome. Int J Urol 8: 495-499, 2001

30. Alexander RB, Ponniah S, Hasday J and Hebel JR: Elevated levels of proinflammatory cytokines in the semen of patients with chronic prostatitis/chronic pelvic pain syndrome. Urology 52: 744-749, 1998.

31. Zhang ZY and Schluesener HJ: HDAC inhibitor MS-275 attenuates the inflammatory reaction in rat experimental autoimmune prostatitis. Prostate 72: 90-99, 2012.

32. Shahed AR and Shoskes DA: Correlation of beta-endorphin and prostaglandin E2 levels in prostatic fluid of patients with chronic prostatitis with diagnosis and treatment response. J Urol 166: $1738-1741,2001$

33. Taguchi O and Nishizuka Y: Self tolerance and localized autoimmunity. Mouse models of autoimmune disease that suggest tissue-specific suppressor T cells are involved in self tolerance. J Exp Med 165: 146-156, 1987.

34. Motrich RD, Maccioni M, Riera CM and Rivero VE: Autoimmune prostatitis: State of the art. Scand J Innunol 66: 217-227, 2007.

35. Pacheco-Rupil B, Depiante-Depaoli $M$ and Casadio B: Experimental autoimmune damage to rat male accessory glands. II. $T$ cell requirement in adoptive transfer of specific tissue damage. Am J Reprod Immunol 5: 15-19, 1984.

36. Ishigooka M, Zermann DH, Doggweiler R and Schmidt RA: Similarity of distributions of spinal c-fos and plasma extravasation after acute chemical irritation of the bladder and the prostate. J Urol 164: 1751-1756, 2000.

37. Quick ML, Wong L, Mukherjee S, Done JD, Schaeffer AJ and Thumbikat P: Th 1-Th 17 cells contribute to the development of uropathogenic Escherichia coli-induced chronic pelvic pain. PLoS One 8: e60987, 2013.

38. Day YJ, Liou JT, Lee CM, Lin YC, Mao CC, Chou AH, Liao CC and Lee HC: Lack of interleukin-17 leads to a modulated micro-environment and amelioration of mechanical hypersensitivity after peripheral nerve injury in mice. Pain 155: 1293-1302, 2014.

39. Murphy SF, Schaeffer AJ, Done J, Wong L, Bell-Cohn A Roman K, Cashy J, Ohlhausen M and Thumbikat P: IL17 Mediates Pelvic Pain in Experimental Autoimmune Prostatitis (EAP). PLoS One 10: e0125623, 2015.

40. Quick ML, Mukherjee S, Rudick CN, Done JD, Schaeffer AJ and Thumbikat P: CCL2 and CCL3 are essential mediators of pelvic pain in experimental autoimmune prostatitis. Am J Physiol Regul Integr Comp Physiol 303: R580-R589, 2012.

41. Rivero VE, Cailleau C, Depiante-Depaoli M, Riera CM and Carnaud C: Non-obese diabetic (NOD) mice are genetically susceptible to experimental autoimmune prostatitis (EAP). J Autoimmun 11: 603-610, 1998. 